\title{
Diffusion-Weighted Imaging of the Head and Neck: Influence of Fat-Suppression Technique and Multishot 2D Navigated Interleaved Acquisitions
}

\author{
(D)Y.J. Bae, DB.S. Choi, (D)H.-K. Jeong, (D) L. Sunwoo, (D) C. Jung, and DJ.H. Kim
}

\begin{abstract}
BACKGROUND AND PURPOSE: DWI of the head and neck can reveal valuable information, but the effects of fat suppression and multishot acquisition on image quality have not been thoroughly investigated. We aimed to comprehensively compare the quality of head and neck DWI at 3T using 2 fat-suppression techniques, STIR, and spectral presaturation with inversion recovery, which were used with both single- and multishot EPI.
\end{abstract}

\begin{abstract}
MATERIALS AND METHODS: Sixty-five study participants underwent 3 DWI sequences of single-shot EPI-STIR, single-shot EPI-spectral presaturation with inversion recovery, and multishot EPI-spectral presaturation with inversion recovery of the head and neck. In multiple anatomic regions, 2 independent readers assessed 5-point visual scores for fat-suppression uniformity and image distortion, and 1 reader measured the contrast-to-noise ratio and ADC.

RESULTS: The mean visual score for fat-suppression uniformity was higher in single-shot EPI-STIR than in other sequences (all regions except for the orbital region, $P<.05$ ). The mean visual score for image distortion was higher in multishot EPI-spectral presaturation with inversion recovery than in single-shot EPI sequences (all regions, $P<.001$ ). Contrast-to-noise ratio was mostly lower in single-shot EPI-STIR than in other sequences $(P<.001)$, and ADC was significantly higher in multishot EPI-spectral presaturation with inversion recovery than in single-shot EPI sequences $(P \leq .001)$.
\end{abstract}

CONCLUSIONS: Overall, multishot EPI-spectral presaturation with inversion recovery provided the best image quality, with relatively homogeneous fat suppression, less image distortion than single-shot EPI sequences, and higher contrast-to-noise ratio than single-shot EPI-STIR. The measured ADC values can be higher in multishot EPI-spectral presaturation with inversion recovery, which necessitates cautious application of the previously reported ADC values to clinical settings.

ABBREVIATIONS: IRIS = image reconstruction using image-space sampling; msEPI = multishot EPI; RESOLVE = readout-segmented EPI using parallel imaging and a $2 \mathrm{D}$ navigator; SPIR = spectral presaturation with inversion recovery; ssEPI = single-shot EPI; CNR = contrast-to-noise ratio

D WI can measure differences in tissue microstructure based on the random displacement of water molecules. ${ }^{1,2}$ The differences in water mobility are quantified by the ADC, which is inversely correlated with tissue cellularity. ${ }^{2,3}$ Using this property, DWI can offer additional information about lesion characteristics in different regions of the body, including the head and neck. ${ }^{2,4-8}$ In clinical practice, DWI is usually performed with a single-

Received February 17, 2017; accepted after revision August 19.

From the Department of Radiology (Y.J.B., B.S.C., L.S., C.J., J.H.K.), Seoul National University Bundang Hospital, Seongnam, Republic of Korea; and Philips Korea (H.-K.J.), Seoul, Republic of Korea.

Please address correspondence to Byung Se Choi, MD, PhD, Department of Radiology, Seoul National University Bundang Hospital, 82, Gumi-ro 173 beon-gil, Bundang-gu, Seongnam, 13620, Republic of Korea; e-mail: byungse.choi@gmail.com

EIndicates article with supplemental on-line appendix and tables.

Indicates article with supplemental on-line photo.

http://dx.doi.org/10.3174/ajnr.A5426 shot EPI (ssEPI) sequence. ${ }^{2,4-8}$ To obtain good ssEPI quality, effective fat suppression is essential to eliminate the lipid signal and reduce ghost artifacts. ${ }^{4}$ Previous studies conducted on breast tissue have demonstrated that uniformity of fat suppression, SNR, contrast-to-noise ratio (CNR), and even ADC are affected by the choice of the fat-suppression technique. ${ }^{4,9-13}$ There has been only 1 study of the head and neck region that evaluated the effect of the fat-suppression technique on ssEPI-DWI. ${ }^{4}$ The authors suggested that STIR offered better image quality, but they assessed the images on a single level using an ROI covering the entire area of the depicted structure, a technique that failed to consider the complex anatomy of the head and neck. ${ }^{4}$ In addition, they did not compare the ADC among different fat-suppression techniques.

Multishot EPI (msEPI) is an imaging technique that reduces magnetic susceptibility artifacts and T2* blurring in DWI., 14-16 Because the head and neck region has substantial magnetic field inhomogeneity due to air-containing structures, the utility of 
msEPI-DWI has been proved effective in decreasing geometric distortion. ${ }^{5,17}$ However, msEPI-DWI requires spectral-selective saturation such as spectral presaturation with inversion recovery (SPIR) for optimal fat suppression, not STIR. ${ }^{15}$ Two studies have compared the image quality between ssEPI-DWI and msEPIDWI in the head and neck, ${ }^{5,17}$ but neither considered fat-suppression techniques.

The aim of this study was to perform a comprehensive comparison of the quality of DWI in the head and neck using different fat-suppression techniques and to determine the utility of the msEPI sequence. Specifically, we adopted 2D navigated interleaved msEPI-DWI with image reconstruction using image-space sampling (IRIS) functions, ${ }^{15,16}$ which has been known to reduce ghosts and off-resonance artifacts but has not yet been used to study the head and neck region. We performed ssEPI-DWI with STIR (ssEPI-STIR), ssEPI-DWI with SPIR (ssEPI-SPIR), and msEPI-DWI with SPIR (msEPI-SPIR) in the head and neck region using a 3T MR imaging scanner. The purpose of our study was to compare both qualitative and quantitative measurements of the image qualities of the above 3 sequences.

\section{MATERIALS AND METHODS \\ Subjects}

The institutional review board of our hospital approved this retrospective study; the requirement for informed consent was waived. Between July and December of 2016, one hundred thirtynine subjects with various pathologies underwent head and neck MR imaging at our institution. We excluded subjects who did not undergo the 3 DWI sequences of interest (ssEPI-STIR, ssEPISPIR, and msEPI-SPIR) ( $n=69)$, and who were younger than 19 years of age $(n=5)$. As a result, 65 subjects ( 43 men and 22 women; age range, 26-85 years; mean age, 57.4 years) were included. The underlying pathologies of the included subjects were as follows: abscess $(n=2)$, dentigerous cyst $(n=1)$, invasive fungal sinusitis and/or skull base osteomyelitis $(n=3)$, inverted papilloma $(n=2)$, lipogranuloma $(n=1)$, metastatic squamous cell carcinoma $(n=2)$, nasal hemangioma $(n=1)$, neurogenic tumor $(n=2)$, squamous papilloma $(n=1)$, parapharyngeal tumor $(n=2)$, parotid tumor $(n=7)$, posttreatment status of cancer including basal cell carcinoma $(n=1)$, lymphoma $(n=3)$, malignant melanoma $(n=1)$, maxillary sinus cancer $(n=2)$, nasal cavity cancer $(n=3)$, nasopharynx cancer $(n=11)$, olfactory neuroblastoma $(n=1)$, oral cavity cancer $(n=11)$, temporal bone metastasis $(n=1)$, and tonsillar cancer $(n=7)$.

\section{MR Imaging Protocol}

MR imaging was performed with a $3 \mathrm{~T}$ instrument (Ingenia; Philips Healthcare, Best, the Netherlands) with a 32-channel sensitivity encoding head coil. DWI was performed in the axial plane using b-values of 0 and $1000 \mathrm{~s} / \mathrm{mm}^{2}$, and 3 orthogonal directions of diffusion gradients. Other imaging parameters for ssEPI-STIR, ssEPI-SPIR, and msEPI-SPIR are summarized in On-line Table 1. Regarding $\mathrm{Z}$-axis scan coverage, we obtained axial images centering the lesion of interest depending on each subject. Therefore, the scan range for the $\mathrm{z}$-axis varied among the subjects. Thus, we did not fully cover the following structures: the orbital region in 15 subjects, the pons in 7 subjects, and the cerebellum in 3 sub- jects. In addition, the larynx was covered in 21 subjects, and the shoulder was covered in 12 subjects, respectively. Besides the DWI, the following sequences were obtained using routine protocols for head and neck MR imaging: axial TSE T2WI with and without fat suppression, axial TSE T1WI, coronal TSE T2WI with fat suppression, and axial/coronal/sagittal TSE post-contrast-enhanced T1WI with fat suppression.

\section{Qualitative Imaging Analysis}

Image quality in relation to the means of fat-suppression uniformity and image distortion was evaluated with a 5-point visual scale. Two board-certified neuroradiologists (Y.J.B. and B.S.C, with 8 and 18 years of experience, respectively), who were blinded to the clinical information and the imaging methods, independently visually inspected the 3 DWI sequences from all study subjects.

Visual scoring for fat-suppression uniformity was as follows: 5 , homogeneous fat suppression; 4 , a few artifacts from insufficient fat suppression; 3 , heterogeneous fat suppression without impairment of lesion analysis; 2 , mostly not suppressed, making lesion identification unfeasible due to ghost artifacts; 1 , no fat suppression. Fat-suppression uniformity was assessed in the anatomic orbital, buccal, and mental regions and the posterior neck and shoulder. A 5-point scale for image distortion was as follows: 5 , excellent image quality with no geometric distortion or susceptibility artifacts; 4 , good image quality with little distortion and few artifacts; 3 , fair image quality, some distortion and artifacts; 2 , poor image quality, with substantial distortion and many artifacts; 1 , unacceptable. The anatomic regions for the assessment of image distortion were the orbit-sphenoethmoid sinus level, posterior fossa, nasal cavity-maxillary sinus level, pharynx, oral cavity, larynx, posterior neck, and shoulder. Due to short scan coverage, the assessment of the orbital region failed in 15 subjects, and the larynx and shoulder were assessed in 21 and 15 subjects.

\section{Quantitative Imaging Analysis}

CNR Calculation. The ROI allocation for quantitative imaging analysis was performed by 1 neuroradiologist (Y.J.B). On ssEPISTIR, ssEPI-SPIR, and msEPI-SPIR with a b-value of $1000 \mathrm{~s} / \mathrm{mm}^{2}$, smoothed polygonal ROIs were drawn in the pons, cerebellar white matter, parotid gland, lymph node, palatine tonsil, semispinalis capitis muscle, and orbital fat, while attempting to include as much of the anatomic structure as possible (On-line Fig 1). The $\mathrm{B}_{0}$ maps of the 3 DWI sequences and the axial T2WIs were used as references to identify the anatomic points for ROI placement. The CNR was calculated using the automatically measured signal intensities in each ROI as follows: CNR $=($ Signal Intensity in Targeted Structure - Signal Intensity in Orbital Fat)/Signal Intensity in Orbital Fat.

Among the study subjects, ROI placement failed in the pons of 7 subjects and in the cerebellum of 3 subjects due to short scan coverage, in the lymph node of 2 subjects due to lack of the presence of a feasible lesion, in the palatine tonsil of 26 subjects due to the small volume of tonsillar tissue or posttreatment status, and in the orbital fat of 15 subjects due to short scan coverage.

ADC Measurement. The above ROIs were copied and pasted 

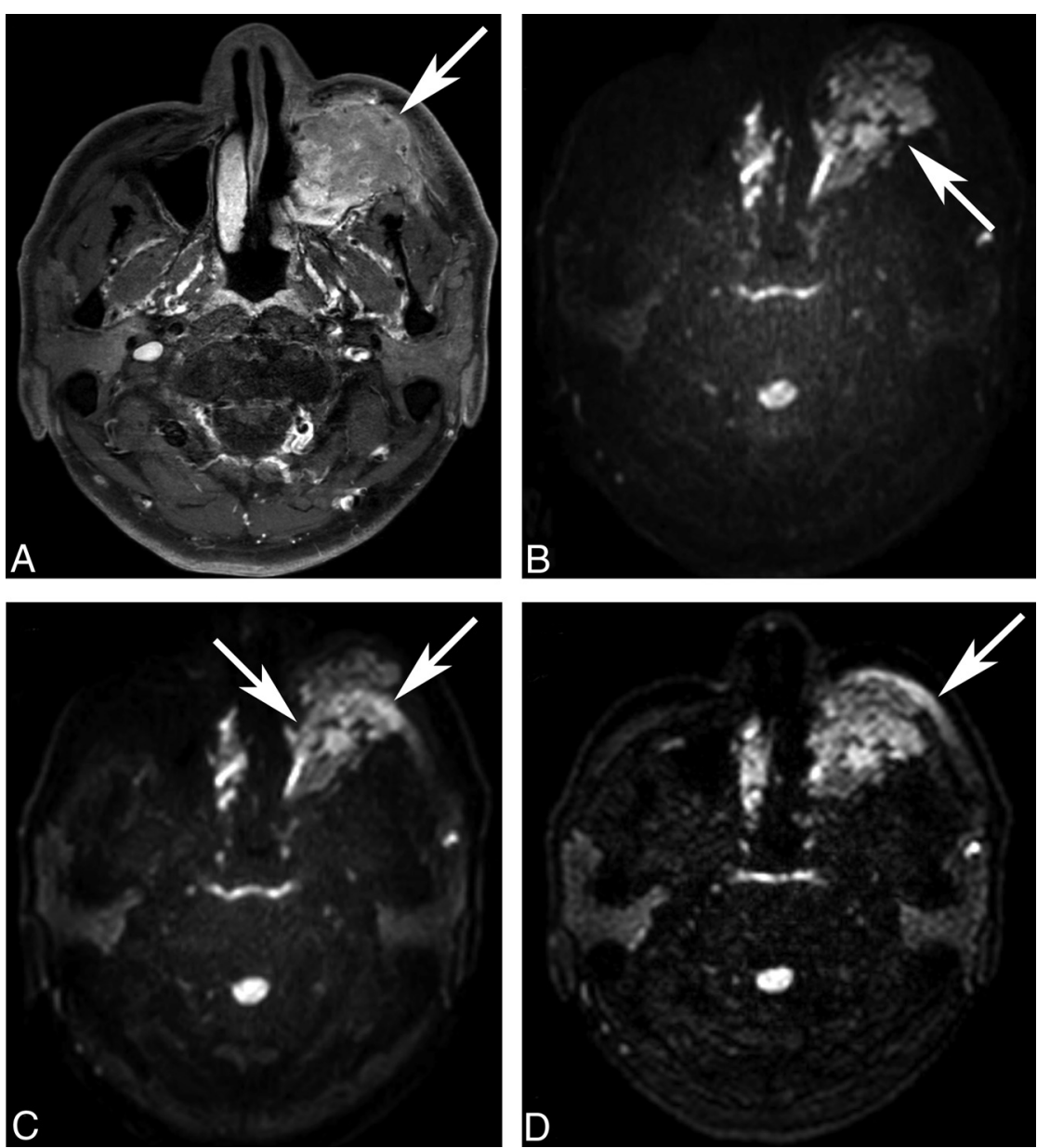

FIG 1. Fat-suppression uniformity of DWI. A 49-year-old man underwent MR imaging for staging of left maxillary sinus cancer ( $A$, post-contrast-enhanced TIWI, arrow). On ssEPI-STIR (B), fat suppression is homogeneous without disrupting the original mass (arrow). On ssEPI-SPIR (C), unsuppressed fat signal (arrows) in the subcutaneous layer creates ghost artifacts that obscure the original mass, making the lesion assessment unfeasible. Unlike ssEPI-SPIR, unsuppressed fat signal (arrow) does not shift much to cover the mass on msEPI-SPIR (D).

onto the corresponding ADC maps from 3 DWI sequences with the same coordinates and areas, excluding orbital fat. The ADC was measured automatically; however, for the same reasons, it was not possible to measure the ADC in the pons of 7 subjects, the cerebellum of 3 subjects, the lymph nodes of 2 subjects, and the palatine tonsil of 26 subjects.

\section{Statistical Analysis}

Continuous variables were expressed as mean \pm SD. $\kappa$ statistics and the $\mathrm{McNemar}$ test were performed to assess the interobserver agreement of the 5-point scale visual scores between the 2 readers. We considered $\kappa$ of $>0.75$ as excellent agreement, $0.40-0.75$ as fair to good, and $<0.40$ as poor. ${ }^{18}$ Visual scores for fat-suppression uniformity and image distortion among the 3 DWI sequences were compared using repeated-measures ANOVA. Then, pair-wise comparison was performed using the paired $t$ test with a Bonferroni correction among 1) ssEPI-STIR and ssEPI-SPIR, 2) ssEPI-STIR and msEPI-SPIR, and 3) ssEPI-SPIR and msEPI-SPIR. The CNR and $\mathrm{ADC}$ of the $3 \mathrm{DWI}$ sequences were compared using repeated-measures ANOVA. The Mauchly test of sphericity was used to test the assumption of the repeated-measures ANOVA, and the Green-
house-Geissler correction method was considered if sphericity was violated. Pairwise comparison was also performed using a paired $t$ test with a Bonferroni correction between each 2 DWI sequences. A $P$ value $<.05$ indicated statistical significance, and for pair-wise comparison, a $P$ value $<.017$ was considered statistically significant based on the Bonferroni correction. All statistical analyses were performed with SPSS software (Version 24.0; IBM, Armonk, New York).

\section{RESULTS \\ Qualitative Assessment}

Reliabilities of visual scores are explained in the On-line Appendix (Online Tables 2 and 3).

Visual scores for fat-suppression uniformity are summarized in On-line Table 4. In both readers, the mean visual score was higher in ssEPI-STIR than in msEPISPIR and ssEPI-SPIR in all regions (all, $P<.05$ ) (Fig 1) except for the orbital region. In the orbital region, all 3 sequences showed excellent fat-suppression uniformity with visual scores higher than 4, which made no significant difference (reader $1, P=.370$; reader $2, P=.363$ ). In the buccal and mental regions, mean scores were significantly higher in msEPISPIR than in ssEPI-SPIR (reader $1, P=$ .005 and $<.001$; reader $2, P=.008$ and $<.001)$. Mean scores in the posterior neck and shoulder were not significantly different between msEPI-SPIR and ssEPI-SPIR in both readers (all, $P>.05$ ).

On-line Table 5 summarizes visual scores for image distortion. Mean visual scores for image distortion were significantly higher in msEPI-SPIR than in ssEPI-STIR and ssEPI-SPIR (both readers, all regions, $P<.001$ ) (Fig 2 ). The mean scores between ssEPISTIR and ssEPI-SPIR showed no significant differences for both readers (all, $P>.05$ ).

\section{Quantitative Assessment}

Quantitative results for CNR and ADC measurements are provided in Table 1.

CNR. CNR was significantly lower in ssEPI-STIR than in ssEPISPIR and msEPI-SPIR in all targeted structures (all, $P<.001$ ). There was no significant difference in CNR between msEPI-SPIR and ssEPI-SPIR in all regions other than the parotid gland where CNR was significantly higher in msEPI-SPIR than in ssEPI-SPIR $(P<.001)$.

ADC. ADC was significantly higher in msEPI-SPIR than in ssEPI-STIR and in ssEPI-SPIR in all targeted structures (all, $P \leq$ $.001)$. In the cerebellar white matter, parotid gland, and semispi- 

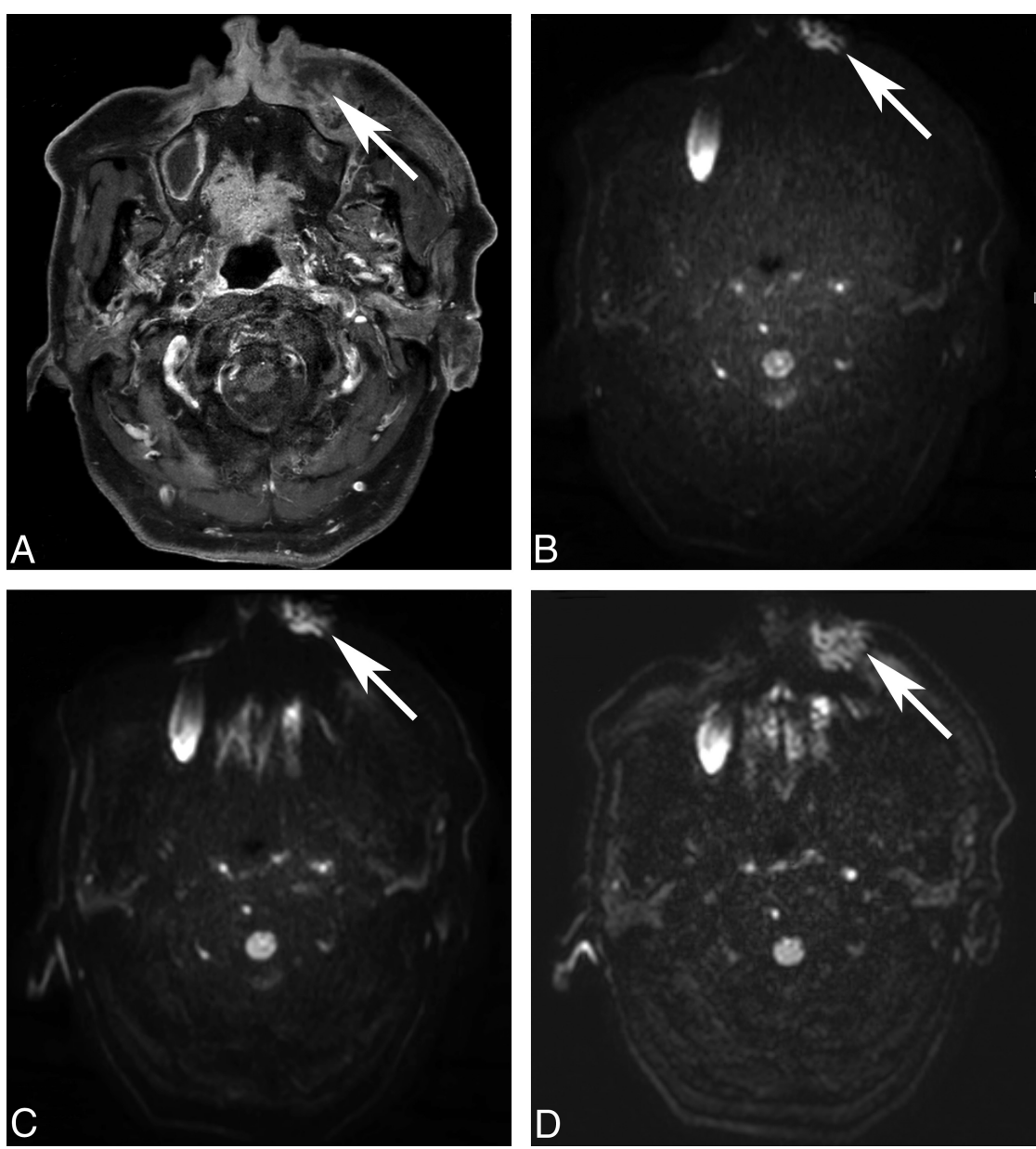

FIG 2. Image distortion of DWI. An 85-year-old man underwent MR imaging for the evaluation of a left premaxillary abscess (A, post-contrast-enhanced TTWI, arrow). Image distortion is increased on ssEPI-STIR ( $B$, arrow) and ssEPI-SPIR (C, arrow) and decreased on msEPI-SPIR ( $D$, arrow). The left premaxillary abscess is not distorted by the susceptibility artifacts only on msEPI-SPIR.

Table 1: Quantitative assessment of DWI

\begin{tabular}{lcccc}
\hline & SsEPI-STIR & ssEPI-SPIR & msEPI-SPIR & $\boldsymbol{P ~ V a l u e s ~}^{\mathbf{b}}$ \\
\hline CNR & & & & \\
$\quad$ Pons & $4.45 \pm 0.84$ & $8.29 \pm 1.62$ & $7.69 \pm 1.90$ & $.001 / .001 / .022$ \\
Cerebellar white matter & $3.86 \pm 0.73$ & $8.78 \pm 1.53$ & $8.52 \pm 2.13$ & $.001 / .001 / .294$ \\
Parotid gland & $0.43 \pm 0.41$ & $1.21 \pm 0.68$ & $1.74 \pm 0.82$ & $.001 / .001 / .001$ \\
Lymph node & $4.55 \pm 1.59$ & $6.61 \pm 2.02$ & $7.06 \pm 2.56$ & $.001 / .001 / .104$ \\
Palatine tonsil & $4.46 \pm 2.47$ & $6.37 \pm 2.27$ & $6.55 \pm 2.12$ & $.001 / .001 / .61$ \\
Semispinalis muscle & $0.40 \pm 0.22$ & $0.87 \pm 0.31$ & $1.00 \pm 0.50$ & $.001 / .001 / .05$ \\
ADC $\left(\times 10^{-3} \mathrm{~mm}^{2} / \mathrm{s}\right)$ & & & & \\
Pons & $0.737 \pm 0.042$ & $0.743 \pm 0.033$ & $0.799 \pm 0.083$ & $.236 / .001 / .001$ \\
Cerebellar white matter & $0.636 \pm 0.039$ & $0.655 \pm 0.030$ & $0.691 \pm 0.061$ & $.001 / .001 / .001$ \\
Parotid gland & $0.771 \pm 0.170$ & $0.867 \pm 0.147$ & $0.907 \pm 0.141$ & $.001 / .001 / .001$ \\
Lymph node & $0.900 \pm 0.321$ & $0.915 \pm 0.349$ & $1.028 \pm 0.341$ & $.300 / .001 / .001$ \\
Palatine tonsil & $0.753 \pm 0.325$ & $0.748 \pm 0.321$ & $0.846 \pm 0.310$ & $.818 / .001 / .001$ \\
Semispinalis muscle & $0.881 \pm 0.187$ & $1.118 \pm 0.115$ & $1.360 \pm 0.107$ & $.001 / .001 / .001$ \\
\hline
\end{tabular}

${ }^{a}$ Data are given as mean $\pm S D$.

${ }^{b} P$ values are derived from pair-wise comparison using a paired $t$ test with a Bonferroni correction among SSEPI-STIR and ssEPI-SPIR/ssEPI-STIR and msEPI-SPIR/ssEPI-SPIR and msEPI-SPIR.

nalis muscle, the ADC was highest in msEPI-SPIR, lower in ssEPISPIR, and lowest in ssEPI-STIR with statistical significance (all, $P \leq .001)$. There was no significant difference in the ADC between ssEPI-STIR and ssEPI-SPIR in the pons, lymph nodes, and tonsil (all, $P>.017$ ).

\section{DISCUSSION}

This study was a comprehensive comparison among 3 DWI sequences, ssEPISTIR, ssEPI-SPIR, and msEPI-SPIR, in the head and neck using both qualitative and quantitative measurements. Our results showed that fat-suppression uniformity was significantly better in ssEPISTIR than in the 2 sequences using SPIR, but image distortion was significantly improved in msEPI-SPIR compared with ssEPI sequences. The CNR was significantly lower in ssEPI-STIR than in msEPI-SPIR and ssEPI-SPIR. The ADC was significantly higher in msEPI-SPIR than ssEPI sequences.

Homogeneous fat suppression in DWI is essential to avoid ghost artifacts. ${ }^{4,19}$ Another concern regarding fat suppression in DWI is that not only visual quality but also SNR, CNR, and even ADC could be altered according to the choice of fat-suppression technique., ${ }^{40-13,19}$ Thus, selecting the optimal fat-suppression technique is a major determinant of the image quality of DWI.

Among fat-suppression techniques, SPIR is widely used on the basis of the frequency-selective radiofrequency pulse that excites only fat spins using chemical shift differences between lipid and water protons. ${ }^{4,10,11,13}$ However, this technique often fails to provide homogeneous fat suppression in regions of the body where field inhomogeneity is substantial. ${ }^{4,10-12,20,21}$ On the other hand, the STIR method uses a nonselective $180^{\circ}$ inversion pulse and the relatively short $\mathrm{T} 1$ relaxation time of the lipid proton to nullify the fat signal. Thus, it is less sensitive to field heterogeneity. ${ }^{4,10-12}$ Therefore, STIR is known to be a more reliable imaging technique for large body parts such as the breast. ${ }^{4,10,11}$

The head and neck region has large susceptibility effects due to air-containing structures. However, few studies have investigated the effects of fat-suppression techniques on the image quality of DWI. In 2014, Maehara et $\mathrm{al}^{4}$ reported that STIR provided good image quality for visual inspection, despite SNR and CNR being higher when the chemical shift selective method, based on frequency-selective pulse, was used. However, this study had several limitations that could hamper its general application. First, the number of study subjects was relatively small $(n=25)$. Second, the authors did not consider the complex 
anatomy of the head and neck; instead, they measured SNR and CNR in 2 ROIs allocated in the semispinalis capitis muscle and the whole area of depicted structures. Third, they did not measure and compare the ADC according to fat-suppression techniques, which could be affected by fat suppression. ${ }^{12,13,21}$

We must also consider msEPI when evaluating the image quality of head and neck DWI. The msEPI technique acquires only a subset of $k$-space samples per each excitation, thereby reducing bandwidth-related EPI artifacts and phase errors. ${ }^{5,15-17}$ Two previous studies have used readout-segmented EPI using parallel imaging and a 2D navigator (RESOLVE) to assess its advantage when applied to head and neck DWI. ${ }^{5,17}$ These studies have discovered that image quality was significantly improved in RESOLVE by reducing susceptibility artifacts, geometric distortion, and blurring, resulting in homogeneous images. ${ }^{5,17}$ Nevertheless, we should consider that msEPI should be coupled with the fat-suppression technique SPIR, not STIR. ${ }^{15,16}$ The use of STIR for each shot on msEPI would cause substantial signal loss or a significant increase in scan time.

Our study was the first to consider both fat suppression and msEPI acquisition when performing a comprehensive comparison of the quality of DWI in the head and neck. In addition, our study has several other advantages. We enrolled by far the largest number of study subjects among head and neck DWI studies, and we assessed both qualitative and quantitative measurements in multiple segmented regions of the head and neck with consideration of anatomic structures. Our study was also the first to adopt the $2 \mathrm{D}$ navigated interleaved msEPI-DWI with IRIS of the head and neck. Similar to RESOLVE, msEPI-DWI with IRIS acquires $2 \mathrm{D}$ navigator echoes to permit further correction of motion-induced phase errors. ${ }^{15,16}$ Moreover, when used in conjunction with parallel imaging methods, an efficient reconstruction method for interleaved msEPI acquisitions (ie, IRIS) was provided to enhance phase correction and achieve high spatial resolution. ${ }^{15,16}$

Our results of the qualitative imaging assessment showed that fat suppression was more homogeneous in ssEPI-STIR than in msEPI-SPIR and ssEPI-SPIR (On-line Table 4). This result agrees with previous studies, which reported that STIR could provide more homogeneous fat suppression in body parts with severe field inhomogeneity than SPIR. ${ }^{4,10-12,19}$ With the same use of SPIR, msEPI-SPIR had a higher visual score for fat-suppression uniformity than ssEPI-SPIR in the buccal and mental regions. We believe that this result was due to the reduction of ghost artifacts from unsuppressed fat signals on the msEPI sequence compared with the ssEPI sequence (Fig 1)..$^{5,15-17}$ Unlike ssEPI-SPIR, the reduction of ghost artifacts could eliminate the obscuring of targeted structures by unsuppressed fat signals on msEPI-SPIR. The difference between msEPI-SPIR and ssEPI-SPIR was not significant in the orbital region and posterior neck where fat suppression was equally excellent and in the shoulder where both sequences failed in homogeneous fat suppression. In the orbital region, all 3 sequences showed comparably excellent fat-suppression homogeneity. More important, regarding image distortion, the distortion was much more reduced in msEPI-SPIR than in ssEPI-STIR and ssEPI-SPIR (On-line Table 5 and Fig 2). Taken together with the above results, we concluded through visual as- sessment that msEPI-SPIR provided the best image quality with relatively homogeneous fat suppression and less image distortion compared with ssEPI-STIR and ssEPI-SPIR, but for imaging the shoulder region, msEPI-SPIR could be more limited than ssEPI-STIR.

In quantitative measurements, CNR was significantly lower in ssEPI-STIR than in msEPI-SPIR and in ssEPI-SPIR, regardless of the anatomic structures (Table 1). With the STIR method, the SNR and CNR are lower than with the spectral-selective presaturation methods because the STIR method uses a nonselective $180^{\circ}$ inversion pulse and most tissues recover more slowly than fat. $^{4,10,12}$ In accordance with previous reports of head and neck DWI,${ }^{5,17}$ no significant difference in CNR between msEPI-SPIR and ssEPI-SPIR was observed in most anatomic structures. However, in the parotid gland, CNR was significantly higher in msEPISPIR than in ssEPI-SPIR. Because our study included a much larger number of study subjects than previous reports and we were the first to use interleaved msEPI-DWI with IRIS in lieu of RESOLVE, the difference in the CNR result may be acceptable with the following explanation: We assumed that the higher CNR in the msEPI sequence was due to the reduction of the blurring effect from T2 decay in the msEPI sequence, which resulted from a smaller echo-train length than in the ssEPI sequence. ${ }^{15,16}$ Along with the qualitative assessment, we could certify that msEPI-SPIR provided the best image quality for head and neck DWI.

The ADC was significantly higher in msEPI-SPIR than in ssEPI-STIR and ssEPI-SPIR. This result also seemed to contradict previous reports, ${ }^{5,17}$ but it may be explained in several ways. First, the STIR method may lead to an underestimation of measured ADC values due to lower SNR than the SPIR method. ${ }^{12,13,21}$ In addition, considering the possibility of a higher CNR of the msEPI-SPIR than of the ssEPI sequence, we could easily assume that the ADC could be higher in msEPI-SPIR than in ssEPI-SPIR. Second, various motion artifacts from cardiac motion, respiratory movement, or swallowing could affect the measurement of ADC values. Our msEPI sequence used a $2 \mathrm{D}$ navigator to correct inplane motion, but it could be affected by through-plane motion along the z-axis. ${ }^{15,16}$ In fact, 1 study applying the msEPI sequence in DWI of the liver ${ }^{22}$ resulted in a higher value of ADC in msEPI than in ssEPI, which is in accordance with our results. Because the head and neck region has a high possibility of motion artifacts, motion artifacts may be the causative factor for determining ADC values. Last, interleaved msEPI-SPIR with IRIS and RESOLVE are applicable in different vendors that use different diffusion gradients. Different imaging gradients can influence the actual b-values and subsequently the ADC, due to so-called cross-term interactions. ${ }^{23}$ As a result, the ADC value could vary depending on the choice of fat-suppression technique and msEPI acquisition, and we should be cautious when applying the previously reported $\mathrm{ADC}$ values to lesion characterization in our own DWI.

Our study has some limitations. First, the number of study subjects whose DWI scans covered the larynx and shoulder was small. Further study with larger numbers of DWI scans including the lower neck area will be needed. Second, the 3 DWI sequences have not been applied in clinical usage such as the characterization between benign and malignant lesions or monitoring responses after cancer treatment. Thus, future studies regarding the 
clinical use of the 3 DWI sequences should be performed to further evaluate their diagnostic values. Third, we used a b-value of $1000 \mathrm{~s} / \mathrm{mm}^{2}$ in our study. To our knowledge, there has been no study regarding the optimal b-value for DWI in the head and neck. Therefore, future studies are needed to verify this issue.

\section{CONCLUSIONS}

Our results indicate that msEPI-SPIR could provide the best image quality with relatively few artifacts from insufficient fat suppression except for the shoulder region, less image distortion than ssEPI sequences, and higher CNR than ssEPI-STIR and/or ssEPISPIR. The measured ADC values can be higher in msEPI-SPIR than in other sequences depending on the effect of the fat-suppression technique used and the msEPI acquisition, which necessitates cautious application of the previously reported ADC values in the individual clinical settings.

\section{ACKNOWLEDGMENTS}

The authors thank the Medical Research Collaborating Center at Seoul National University Bundang Hospital for consultation on the statistical analyses.

Disclosures: Ha-Kyu Jeong—UNRELATED: Employment: Philips Korea, Comments: regular salary.

\section{REFERENCES}

1. Le Bihan D, Breton E, Lallemand D, et al. Separation of diffusion and perfusion in intravoxel incoherent motion MR imaging. Radiology 1988;168:497-505 CrossRef Medline

2. Vandecaveye V, De Keyzer F, Vander Poorten V, et al. Head and neck squamous cell carcinoma: value of diffusion-weighted MR imaging for nodal staging. Radiology 2009;251:134-46 CrossRef Medline

3. Herneth AM, Guccione S, Bednarski M. Apparent diffusion coefficient: a quantitative parameter for in vivo tumor characterization. Eur J Radiol 2003;45:208 -13 CrossRef Medline

4. Maehara M, Ikeda K, Kurokawa H, et al. Diffusion-weighted echoplanar imaging of the head and neck using 3-T MRI: investigation into the usefulness of liquid perfluorocarbon pads and choice of optimal fat suppression method. Magn Reson Imaging 2014;32: 440-45 CrossRef Medline

5. Zhao M, Liu Z, Sha Y, et al. Readout-segmented echo-planar imaging in the evaluation of sinonasal lesions: a comprehensive comparison of image quality in single-shot echo-planar imaging. Magn Reson Imaging 2016;34:166-72 CrossRef Medline

6. Thoeny HC, De Keyzer F, King AD. Diffusion-weighted MR imaging in the head and neck. Radiology 2012;263:19-32 CrossRef Medline

7. Srinivasan A, Dvorak R, Perni K, et al. Differentiation of benign and malignant pathology in the head and neck using $3 \mathrm{~T}$ apparent diffusion coefficient values: early experience. AJNR Am J Neuroradiol 2008;29:40-44 CrossRef Medline

8. Abdel Razek AA, Kandeel AY, Soliman N, et al. Role of diffusion- weighted echo-planar MR imaging in differentiation of residual or recurrent head and neck tumors and posttreatment changes. AJNR Am J Neuroradiol 2007;28:1146-52 CrossRef Medline

9. Woodhams R, Ramadan S, Stanwell P, et al. Diffusion-weighted imaging of the breast: principles and clinical applications. Radiographics 2011;31:1059-84 CrossRef Medline

10. Mürtz P, Tsesarskiy M, Kowal A, et al. Diffusion-weighted magnetic resonance imaging of breast lesions: the influence of different fatsuppression techniques on quantitative measurements and their reproducibility. Eur Radiol 2014;24:2540-51 CrossRef Medline

11. Brandão S, Nogueira L, Matos E, et al. Fat suppression techniques (STIR vs. SPAIR) on diffusion-weighted imaging of breast lesions at 3.0 T: preliminary experience. Radiol Med 2015;120:705-13 CrossRef Medline

12. Baron $P$, Dorrius MD, Kappert $P$, et al. Diffusion-weighted imaging of normal fibroglandular breast tissue: influence of microperfusion and fat suppression technique on the apparent diffusion coefficient. NMR Biomed 2010;23:399-405 CrossRef Medline

13. Wenkel E, Geppert C, Schulz-Wendtland R, et al. Diffusion weighted imaging in breast MRI: comparison of two different pulse sequences. Acad Radiol 2007;14:1077-83 CrossRef Medline

14. Porter DA, Heidemann RM. High resolution diffusion-weighted imaging using readout-segmented echo-planar imaging, parallel imaging and a two-dimensional navigator-based reacquisition. Magn Reson Med 2009;62:468-75 CrossRef Medline

15. Jeong HK, Dewey BE, Hirtle JA, et al. Improved diffusion tensor imaging of the optic nerve using multishot two-dimensional navigated acquisitions. Magn Reson Med 2015;74:953-63 CrossRef Medline

16. Jeong HK, Gore JC, Anderson AW. High-resolution human diffusion tensor imaging using 2-D navigated multishot SENSE EPI at 7 T. Magn Reson Med 2013;69:793-802 CrossRef Medline

17. Koyasu S, Iima M, Umeoka S, et al. The clinical utility of reduceddistortion readout-segmented echo-planar imaging in the head and neck region: initial experience. Eur Radiol 2014;24:3088-96 CrossRef Medline

18. Fleiss JL. Statistical Methods for Rates and Proportions. 2nd ed. New York: John Wiley; 1981

19. Kazama T, Nasu K, Kuroki Y, et al. Comparison of diffusionweighted images using short inversion time inversion recovery or chemical shift selective pulse as fat suppression in patients with breast cancer. Jpn J Radiol 2009;27:163-67 CrossRef Medline

20. Koç O, Paksoy Y, Erayman I, et al. Role of diffusion weighted MR in the discrimination diagnosis of the cystic and/or necrotic head and neck lesions. Eur J Radiol 2007;62:205-13 CrossRef Medline

21. Nakatsu M, Hatabu H, Itoh $\mathrm{H}$, et al. Comparison of short inversion time inversion recovery (STIR) and fat-saturated (chemsat) techniques for background fat intensity suppression in cervical and thoracic MR imaging. J Magn Reson Imaging 2000;11:56-60 Medline

22. Deng J, Miller FH, Salem R, et al. Multishot diffusion-weighted PROPELLER magnetic resonance imaging of the abdomen. Invest Radiol 2006;41:769-75 CrossRef Medline

23. Brihuega-Moreno O, Heese FP, Tejos C, et al. Effects of, and corrections for, cross-term interactions in Q-space MRI. Magn Reson Med 2004;51:1048-54 CrossRef Medline 